\title{
The incidence of symptomatic thrombus in TKA patients has no correlation with use of anticoagulants after discharge
}

\author{
Xiangji Dang ${ }^{1}$, Zhilong Liu ${ }^{2}$, Xiaoyun Sheng ${ }^{3}$, Yan Liu ${ }^{4}$, and Bin Geng ${ }^{5}$ \\ ${ }^{1}$ Department of pharmaceutical, Lanzhou University Second Hospital, Cui Ying Men \\ No.80, Lanzhou 730030, Gansu province, PR China, dangxj1986@163.com \\ ${ }^{2}$ Department of anesthesiology, Gansu Provincial Hospital, Donggang West Road No. 204, \\ Lanzhou 730030, Gansu province, PR China, liuzhilong85@163.com \\ ${ }^{3}$ Department of orthopaedics, Lanzhou University Second Hospital, Cui Ying Men No.80, \\ Lanzhou 730030, Gansu province, PR China, shengxy0310@163.com \\ ${ }^{4}$ Affiliation not available \\ ${ }^{5}$ Department of orthopaedics, Lanzhou University Second Hospital, Cui Ying Men No.80, \\ Lanzhou 730030, Gansu province, PR China, cxxxf@foxmail.com
}

May 6, 2020

\begin{abstract}
Background: Many guidelines indicated that to continue using anticoagulant drugs to reduce the incidence of symptomatic venous thrombosis (SVT) in total knee arthroplasty (TKA) patients after discharged. Objective: This retrospective analysis included 407 consecutive patients who underwent TKA at the Lanzhou University Second Hospital in China. Cases history from Jan. 2013 to Dec. 2016. Methods: The group A were taken hypodermic injection of low molecular heparin for 5-10 days after surgery at the hospitalization time, then continued oral rivaroxaban after hospital discharge. The group B were only taken hypodermic injection of low molecular heparin for 5-10 days at the hospitalization time. Baseline characteristics of patients, total complications of SVT, any venous thromboembolic disease (VTE), deep venous thrombosis (DVT), pulmonary embolism (PE), lower limbs swelling, bleeding and mortality following hospital discharge were compared between the two groups. Main outcome measures: The main outcome measures were incidence of bleeding and death duration of in-hospital and out-hospital stay in this study. Results: After analysis, the results showed that the incidence of SVT patients had no significant difference between the two groups. Moreover, the incidence of VTE, major/minor bleeding, total-DVT, PE, fatal PE and death all had no significant differences between the two groups. But the patients of no smoking or no drinking were superior than the smoking or drinking in the incidence of symptomatic thrombosis. Conclusion: There is no enough evidence showed that the TKA patients given anticoagulant after hospital discharged had beneficial to decrease the risk of SVT incidence. Furthermore, the smoking and drinking would increase the risk of symptomatic thrombosis for TKA patients.
\end{abstract}

\section{Hosted file}

Manuscript-\begin\{CJK\}\{UTF8\}\{gbsn\} \end\{CJK\}\selectlanguage\{english\}2.doc available at } https://authorea.com/users/318287/articles/448221-the-incidence-of-symptomatic-thrombusin-tka-patients-has-no-correlation-with-use-of-anticoagulants-after-discharge

\section{Hosted file}

Tab 1 Baseline Characteristics of the 410Patients in the Safety Population.docx available at https ://authorea. com/users/318287/articles/448221-the-incidence-of-symptomatic-thrombusin-tka-patients-has-no-correlation-with-use-of-anticoagulants-after-discharge 


\section{Hosted file}

Tab2 Efficacy outcomes during treatment period.docx available at https://authorea.com/users/ 318287/articles/448221-the-incidence-of-symptomatic-thrombus-in-tka-patients-has-nocorrelation-with-use-of-anticoagulants-after-discharge

\section{Hosted file}

Tab3 Efficacy and Clinical End Points.docx available at https://authorea.com/users/ 318287/articles/448221-the-incidence-of-symptomatic-thrombus-in-tka-patients-has-nocorrelation-with-use-of-anticoagulants-after-discharge

\section{Hosted file}

Tab4 Bleeding events during the treatment period.docx available at https://authorea.com/ users/318287/articles/448221-the-incidence-of-symptomatic-thrombus-in-tka-patients-hasno-correlation-with-use-of-anticoagulants-after-discharge 